九州大学学術情報リポジトリ

Kyushu University Institutional Repository

\title{
Configurational entropy and heat capacity in supercooled liquids
}

Yoshimori, Akira

Department of Physics, Kyushu University

Odagaki, Takashi

Division of Science, School of Science and Engineering, Tokyo Denki University

http://hdl. hand le. net/2324/25505

出版情報：Journal of the Physical Society of Japan. 80 (6)，pp.064601(1)-064601(5)，2011-0610. Physical Society of Japan

バージョン :

権利関係: (C) 2011 The Physical Society of Japan 


\title{
Configurational Entropy and Heat Capacity in Supercooled Liquids
}

\author{
Akira Yoshimori* and Takashi Odagaki ${ }^{1}$ \\ Department of Physics, Kyushu University, Fukuoka 812-8581, Japan \\ ${ }^{1}$ Division of Science, School of Science and Engineering, Tokyo Denki University, Hatoyama, \\ Hikigun, Saitama 350-0394, Japan
}

\begin{abstract}
We establish the general relationship between the configurational entropy and heat capacity in a supercooled liquid within the framework of equilibrium statistical mechanics. The relationship shows that the configurational entropy is different from the entropy obtained by the integration of the glass heat capacity. In addition, the difference shows the correlation between the energy and entropy in liquid structures. Applying the general results to a simple model, we demonstrate that the difference can be too large to neglect. We also discuss new experiments using the present results.
\end{abstract}

KEYWORDS: configurational entropy, supercooled liquids, thermodynamics by division of degrees of freedom, Einstein oscillators, free energy landscape

\section{Introduction}

Configurational entropy is in a unique position among studies of supercooled liquids and glass transitions because it is related to thermodynamics and structures in liquids. To understand glass transitions, the motions in a supercooled liquid have often been divided into structural relaxation and fine vibrations. ${ }^{1-3}$ The configurational entropy is the contribution from structural relaxation to the thermodynamic entropy. In addition, it expresses the number of stable structures in a liquid system. The configurational entropy has been studied by many authors because it is a key to understanding glass transitions. ${ }^{3-10}$

The configurational entropy is also related to structural relaxation through the cooperative rearrangement region (CRR), which is also an important concept in glass transitions. Adam and Gibbs have considered that structural relaxation is caused by the rearrangement of liquid particles in the CRR. ${ }^{11}$ Using the concept of the CRR, they described the Vogel-Fulcher temperature dependence of the time scale. The configurational entropy was related to the CRR by the assumption that the number of particles included in the CRR (CRR size) is proportional to the inverse of the configurational entropy. Thus, they expressed the time scale of structural relaxation using the configurational entropy. ${ }^{11}$

To obtain the configurational entropy and the CRR size, Yamamuro et al. have proposed an experimental method using heat capacities. ${ }^{4}$ By their pioneered study of the configurational

\footnotetext{
*E-mail address: a.yoshimori@cmt.phys.kyushu-u.ac.jp
} 
entropy, they obtained the entropy, integrating the difference between the heat capacity of supercooled liquids and the interpolated heat capacity of glass. Assuming that the CRR includes only one particle at the high temperature limit, they also obtained 4 to 8 particles in the CRR at glass transition temperatures. Similar values have been obtained with recent experiments on room-temperature ionic liquids. ${ }^{7}$

A theoretical method of obtaining the CRR size has also been proposed. Yoshidome et al. have calculated the CRR size in hard-sphere liquids using the free energy landscape (FEL) theory. ${ }^{12}$ Defining the CRR size as the minimal size giving two basins on the FEL, they calculated the FEL of random-configurational particles on the basis of the density functional theory. They examined the minimal size, moving a particle inside a spherical shell formed by fixing particles.

The theoretical calculations showed that the CRR size was markedly different from the experimental values. Yoshidome et al. obtained more than 20 particles at $\rho \sigma^{3}>1.0$, where $\rho$ is the density and $\sigma$ is the diameter of the hard sphere. ${ }^{12}$ One can attribute the discrepancy to the difference between microscopic interactions in systems employed by the theoretical calculation and experiments. For the theoretical calculation, the hard-sphere system was employed, although the experiments dealt with molecular systems. Using the same method as that used in the experiments, however, molecular dynamics simulations of soft-sphere systems have given similar results to the experiments. ${ }^{13}$

One requires a general theory of the configurational entropy and heat capacity to explain the discrepancy between experimental and theoretical methods. On the basis of the theory, the relationship between configurational entropy and heat capacity, in particular, should be established because it is a basis for the proposed experimental method. It is better to formulate the theory as general as possible, because the derived relationship is restricted if one has assumptions.

The purpose of the present study is to establish the relationship between configurational entropy and heat capacity with as less assumptions as possible. We assume that the degrees of freedom in a system can be divided. Although supercooled liquids are in nonequilibrium, the equilibrium statistical mechanics is assumed as the first step. Results obtained by the general formulation are applied to a simple model to demonstrate the usefulness of the formulation.

\section{General Formulation}

In the present study, we divide all degrees of freedom into two groups, namely, $\left\{x_{1}, x_{2}, \cdots\right\}=\left\{x_{i}\right\}$ and $\left\{X_{1}, X_{2}, \cdots\right\}=\left\{X_{i}\right\}$, considering a three-dimensional $N$-particle system. The two groups do not include the momentum of particles. Such division is useful in studying suppercooled liquids and glass transitions, as is shown by the free energy landscape (FEL) theory ${ }^{2,3,12,14-17}$ and so on. The two groups correspond to fast vibrational motions and structural relaxation in a liquid, when the FEL theory is applied. The present formulation, 
however, does not depend on the way of dividing the degrees of freedom.

We define averages over only one group in the divided degrees of freedom. The average for a physical quantity $A\left(\left\{x_{i}, X_{i}, p_{l}\right\}\right)$ over the degrees of freedom $\left\{x_{i}\right\}$ is defined by

$$
A\left(\left\{X_{i}\right\}\right)=\frac{1}{Z\left(\left\{X_{i}\right\}\right)} \int A\left(\left\{x_{i}, X_{i}, p_{l}\right\}\right) \frac{e^{-\beta H\left(\left\{x_{i}, X_{i}, p_{l}\right\}\right)}}{N ! h^{3 N}} \prod_{i l} d x_{i} d p_{l},
$$

with

$$
Z\left(\left\{X_{i}\right\}\right)=\int \frac{e^{-\beta H\left(\left\{x_{i}, X_{i}, p_{l}\right\}\right)}}{N ! h^{3 N}} \prod_{i l} d x_{i} d p_{l} .
$$

Here, $p_{l}$ is the momentum in degrees of freedom $l$ including the conjugate momentum of $\left\{x_{i}\right\}$ and $\left\{X_{i}\right\}$, and $H\left(\left\{x_{i}, X_{i}, p_{l}\right\}\right)$ is the Hamiltonian of the particle system. In addition, $\beta=1 /\left(k_{B} T\right)$, where $k_{B}$ is the Boltzmann constant and $T$ is the temperature. If $A\left(\left\{x_{i}, X_{i}, p_{l}\right\}\right)$ is the Hamiltonian $H\left(\left\{x_{i}, X_{i}, p_{i}\right\}\right)$, then $A\left(\left\{X_{i}\right\}\right)$ is the energy $E\left(\left\{X_{i}\right\}\right)$.

We define the $\left\{X_{i}\right\}$-dependent free energy, entropy, and probability. Using the partial partition function $Z\left(\left\{X_{i}\right\}\right)$ in eq. (2) as a function of $\left\{X_{i}\right\}$, the free energy $\Phi\left(\left\{X_{i}\right\}\right)$ is defined by

$$
\Phi\left(\left\{X_{i}\right\}\right)=-k_{B} T \ln Z\left(\left\{X_{i}\right\}\right) .
$$

From the free energy $\Phi\left(\left\{X_{i}\right\}\right)$, we define the $\left\{X_{i}\right\}$-dependent entropy by

$$
S\left(\left\{X_{i}\right\}\right)=-\frac{\partial \Phi\left(\left\{X_{i}\right\}\right)}{\partial T} .
$$

The free energy $\Phi\left(\left\{X_{i}\right\}\right)$ also gives the probability $P\left(\left\{X_{i}\right\}\right)$ that the system is found at the state indicated by $\left\{X_{i}\right\}$ :

$$
P\left(\left\{X_{i}\right\}\right)=\frac{\exp \left[-\beta \Phi\left(\left\{X_{i}\right\}\right)\right]}{Z},
$$

where $Z$ is the partition function in the entire system. From this probability, one can average any function of $\left\{X_{i}\right\}, f\left(\left\{X_{i}\right\}\right)$ by

$$
\left\langle f\left(\left\{X_{i}\right\}\right)\right\rangle=\int f\left(\left\{X_{i}\right\}\right) P\left(\left\{X_{i}\right\}\right) \prod_{i} d X_{i} .
$$

If degrees of freedom are divided into fine vibrations and structural relaxation, we define these $X_{i}$-dependent quantities, averaging over fine vibrations with the condition that a liquid structure is fixed. Then, the value of $A\left(\left\{X_{i}\right\}\right)$ changes with the relaxation of the structure, though it does not change only with slight vibrations of liquid particles. We define the $\left\{X_{i}\right\}$-dependent free energy and entropy by fixing the special structure indicated by $X_{i}$. The probability $P\left(\left\{X_{i}\right\}\right)$ expresses the probability that a liquid system has the structure.

Using the $\left\{X_{i}\right\}$-dependent probability, the configurational entropy $S_{c}$ is defined by

$$
S_{c}=-k_{B}\left\langle\ln P\left(\left\{X_{i}\right\}\right)\right\rangle .
$$

Since the definition does not depend on the way of dividing the degrees of freedom, it includes 
various definitions employed for formulation of previous theories, such as the FEL ${ }^{2,3,12,14-17}$ and replica ${ }^{8-10}$ theories. The definition also agrees with $k_{B} \ln W$, where $W$ is the number of states defined by $W=\left\langle\delta\left(\bar{\Phi}-\Phi\left(\left\{X_{i}\right\}\right)\right)\right\rangle$ with $\bar{\Phi}=\left\langle\Phi\left(\left\{X_{i}\right\}\right)\right\rangle$ at the thermodynamic limit.

If two groups in the degrees of freedom are appropriately chosen, the present definition of the configurational entropy leads to the number of stable structures in a liquid. The choice of two groups is given by the fast vibrations and structural relaxation in a liquid. If the degrees of freedom are divided in such a way, discrete stable structures are given by minima of $\Phi\left(\left\{X_{i}\right\}\right)$. From the definition given by eq. (7), if the number of stable structures is denoted by $W$, the thermodynamic limit gives $S_{c}=k_{B} \ln W$.

By the definition of the configurational entropy, one finds that the sum of the configurational and glass entropies expresses the total entropy. To obtain the expression, using eq. (5), we rewrite eq. (7) in the form ${ }^{3}$

$$
S_{c}=\frac{-F+\left\langle\Phi\left(\left\{X_{i}\right\}\right)\right\rangle}{T},
$$

where the Helmholtz free energy $F$ is defined by $-k_{B} T \ln Z$. The total entropy $S$ is related to the free energy $F$ by $F=E-T S$, where $E$ is the total energy. The substitution of the expression with $\Phi\left(\left\{X_{i}\right\}\right)=E\left(\left\{X_{i}\right\}\right)-T S\left(\left\{X_{i}\right\}\right)$ yields

$$
S=S_{c}+\left\langle S\left(\left\{X_{i}\right\}\right)\right\rangle
$$

when $E=\left\langle E\left(\left\{X_{i}\right\}\right)\right\rangle$ is employed. The glass entropy $S_{g}$ is given by the second term in eq. (9) $\left\langle S\left(\left\{X_{i}\right\}\right)\right\rangle$, because it can be interpreted in terms of the quenched average if a glass state is caused by frozen motions of $\left\{X_{i}\right\}$.

Differentiating the expression of the entropy with respect to the temperature, we establish the relationship between configurational entropy and heat capacity. If eq. (9) is differentiated with respect to the temperature, we obtain ${ }^{3}$

$$
C=T \frac{\partial S_{c}}{\partial T}+\left\langle T \frac{\partial S\left(\left\{X_{i}\right\}\right)}{\partial T}\right\rangle+\int T S\left(\left\{X_{i}\right\}\right) \frac{\partial P\left(\left\{X_{i}\right\}\right)}{\partial T} \prod_{i} d X_{i}
$$

where $C$ is the total heat capacity. The first term on the right-hand side of eq. (10) is rewritten using $E=\partial \beta F / \partial \beta=\langle E\rangle=\left\langle\partial \beta \Phi\left(\left\{X_{i}\right\}\right) / \partial \beta\right\rangle$ in the form

$$
T \frac{\partial S_{c}}{\partial T}=\frac{1}{k_{B} T^{2}}\left\langle\Delta \Phi\left(\left\{X_{i}\right\}\right) \Delta E\left(\left\{X_{i}\right\}\right)\right\rangle,
$$

where $\Delta \Phi\left(\left\{X_{i}\right\}\right)=\Phi\left(\left\{X_{i}\right\}\right)-\left\langle\Phi\left(\left\{X_{i}\right\}\right)\right\rangle$ and $\Delta E\left(\left\{X_{i}\right\}\right)=E\left(\left\{X_{i}\right\}\right)-\left\langle E\left(\left\{X_{i}\right\}\right)\right\rangle$. If the glass state is interpreted in terms of frozen motions of $\left\{X_{i}\right\}$, the second term in eq. (10) $\left\langle T \partial S\left(\left\{X_{i}\right\}\right) / \partial T\right\rangle$ can be regarded as the heat capacity of glass. ${ }^{18-22}$ Then, we define

$$
C_{g}=\left\langle T \frac{\partial S\left(\left\{X_{i}\right\}\right)}{\partial T}\right\rangle \text {. }
$$


By using eq. (5), the third term in eq. (10) is reduced to

$$
\int T S\left(\left\{X_{i}\right\}\right) \frac{\partial P\left(\left\{X_{i}\right\}\right)}{\partial T} \prod_{i} d X_{i}=\beta\left\langle\Delta S\left(\left\{X_{i}\right\}\right) \Delta E\left(\left\{X_{i}\right\}\right)\right\rangle,
$$

where $\Delta S\left(\left\{X_{i}\right\}\right)=S\left(\left\{X_{i}\right\}\right)-\left\langle S\left(\left\{X_{i}\right\}\right)\right\rangle$. Here, $\partial\left(\beta \Phi\left(\left\{X_{i}\right\}\right)\right) / \partial \beta=E\left(\left\{X_{i}\right\}\right)$ is employed.

The obtained relationship between configurational entropy and heat capacity shows that the configurational entropy cannot exactly be obtained using the heat capacity. Using the heat capacity of glass defined by eq. (12), we define the following new entropy obtained by the heat capacity: ${ }^{3}$

$$
S_{h}=S-\int_{0}^{T} \frac{C_{g}}{T^{\prime}} d T^{\prime}
$$

From eqs. (10) and (13), one can obtain

$$
S_{c}=S_{h}-\int_{0}^{T} \frac{\left\langle\Delta S\left(\left\{X_{i}\right\}\right) \Delta E\left(\left\{X_{i}\right\}\right)\right\rangle}{k_{B} T^{\prime 2}} d T^{\prime}
$$

Here, we assume $S=S_{c}=0$ at $T \rightarrow 0$. Equation (15) is the main result of the present study.

While the configurational entropy is different from the entropy obtained from the heat capacity, the difference is related to thermodynamic characters in liquid structures. Equation (15) connects the difference with the correlation between the $X_{i}$-dependent energy and the $X_{i^{-}}$ dependent entropy. If $\left\{X_{i}\right\}$ are assumed to be the degrees of freedom expressing the structures, the difference is given by the correlation between the energy and entropy of the structures. For example, the difference $S_{c}-S_{h}$ is negative when low-energy structures tend to have a low entropy at all temperatures. In contrast, the difference $S_{c}-S_{h}$ is positive when low-energy structures tend to have a high entropy.

\section{Application to a Simple Model}

We apply the general formulation presented in the previous section to a simple model to demonstrate the usefulness of the formulation. Using the simple model, we can show numerical values of the difference between $S_{c}$ and $S_{h}$. The model also allows one to consider in details how the difference is caused.

The model consists of $M$ independent elements, among which element $a$ has the variable $x_{a}$ expressing vibrations of particles and the variable $X_{a}$ expressing structural relaxation. An element corresponds to a particle or a group of particles. An element also has a quantum mechanical harmonic oscillator; the Hamiltonian $H$ in the model is given by

$$
H=K+\sum_{a=1}^{M}\left\{u\left(X_{a}\right)+\frac{1}{2} m \omega\left(X_{a}\right) x_{a}^{2}\right\} .
$$

Here, $K$ is the kinetic energy, $u\left(X_{a}\right)$ is the potential energy of $X_{a}, m$ is the mass of the oscillator, and $\omega\left(X_{a}\right)$ is the $X_{a}$-dependent frequency of the oscillator. Assuming that $u\left(X_{a}\right)$ has two deep minima, we consider only two values of $X_{a}$ denoted by $X_{1}^{*}$ and $X_{2}^{*}$, so that only two values of the frequency denoted by $\omega_{1}=\omega\left(X_{1}^{*}\right)$ and $\omega_{2}=\omega\left(X_{2}^{*}\right)$ are also considered. 
Using the model, to obtain the $X_{a}$-dependent thermodynamic quantities defined in the previous section, we calculate the $X_{a}$-dependent free energy, energy, and entropy per element. When the $X_{a}$-dependent free energy per element is denoted by $\phi\left(X_{a}\right)$, the values at the two minima are denoted by $\phi_{1}=\phi\left(X_{1}^{*}\right)$ and $\phi_{2}=\phi\left(X_{2}^{*}\right)$. If $X_{a}$ is fixed at $X_{1}^{*}$ or $X_{2}^{*}$, one has to only calculate the one-quantum oscillator system with the frequency $\omega_{i}(i=1,2)$; we obtain

$$
\phi_{i}=-k_{B} T \ln z_{i}
$$

with

$$
z_{i}=\frac{\exp \left[-\beta\left(\frac{\hbar \omega_{i}}{2}+\epsilon_{i}\right)\right]}{1-\exp [-\beta \hbar \omega]} .
$$

Here, $\hbar$ is the Planck constant divided by $2 \pi$, and $\epsilon_{i}=u\left(X_{i}^{*}\right)$. The $X_{a}$-dependent energy and entropy per element are given in the same way by

$$
\begin{aligned}
e_{i} & =\frac{\hbar \omega_{i}}{\exp \left[\beta \hbar \omega_{i}\right]-1}+\frac{\hbar \omega_{i}}{2}+\epsilon_{i}, \\
s_{i} & =k_{B} \ln z_{i}+\frac{e_{i}}{T}
\end{aligned}
$$

From these quantities, we calculate the entropies by

$$
\begin{aligned}
S & =k_{B} M \ln \left(z_{1}+z_{2}\right)+M \frac{\left\langle e_{i}\right\rangle}{T}, \\
S_{g} & =M\left\langle s_{i}\right\rangle, \\
S_{c} & =-k_{B} M\left\langle\ln P_{i}\right\rangle,
\end{aligned}
$$

where $\left\langle a_{i}\right\rangle=P_{1} a_{1}+P_{2} a_{2}$ and $P_{i}=z_{i} /\left(z_{1}+z_{2}\right)$. From the equations, one can show the general relation $S=S_{g}+S_{c}$ given by eq. (9). Figure 1 shows the temperature dependence of the entropies calculated from the equations when $\hbar \omega_{2}=2 \hbar \omega_{1}$ and $\epsilon_{2}-\epsilon_{1}=-\hbar \omega_{1}$. Although $S$ and $S_{g}$ increased with temperature, $S_{c}$ approached a constant value at high temperatures.

From the entropies, one can obtain the heat capacities

$$
C=C_{c}+C_{g}+\Delta C
$$

where the first term $C_{c}$ is obtained from eq. (11) by

$$
C_{c}=T \frac{\partial S_{c}}{\partial T}=\frac{M}{k_{B} T^{2}}\left\langle\Delta \phi_{i} \Delta e_{i}\right\rangle .
$$

We have $C_{g}$ from eq. (19) or (20) and $\Delta C$ from eq. (13) by

$$
\begin{aligned}
C_{g} & =M k_{B}\left\langle\frac{\left(\beta \hbar \omega_{i}\right)^{2} e^{\beta \hbar \omega_{i}}}{e^{\beta \hbar \omega_{i}}-1}\right\rangle, \\
\Delta C & =\beta M\left\langle\Delta s_{i} \Delta e_{i}\right\rangle,
\end{aligned}
$$

with $\Delta \phi_{i}=\phi_{i}-\left\langle\phi_{i}\right\rangle$ and so on. Figure 2 shows the temperature dependence of these heat capacities when $\hbar \omega_{2}=2 \hbar \omega_{1}$ and $\epsilon_{2}-\epsilon_{1}=-\hbar \omega_{1}$. As temperature increased, $C_{c}$ and $\Delta C$ 


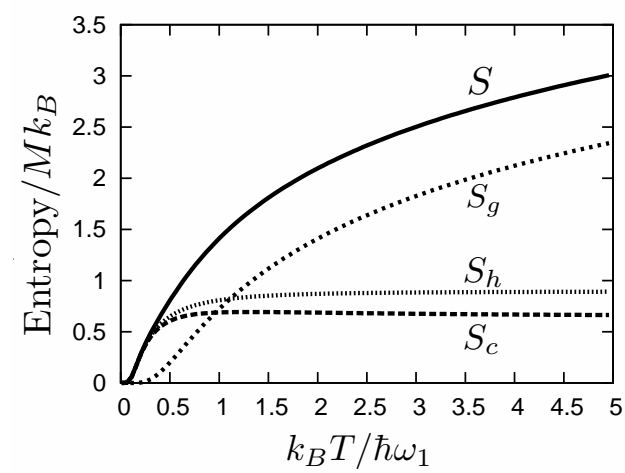

Fig. 1. Calculated temperature dependence of the total entropy $S$, glass entropy $S_{g}$, configurational entropy $S_{c}$, and entropy obtained from the glass heat capacity by eq. (14) $S_{h}$. The model employed for the calculation consists of $M$ independent elements, each of which takes either of the two quantum harmonic oscillators. The ratio of the frequencies is given by $\omega_{2} / \omega_{1}=2$. The difference in the potential bottoms is given by $\epsilon_{2}-\epsilon_{1}=-\hbar \omega_{1}$.

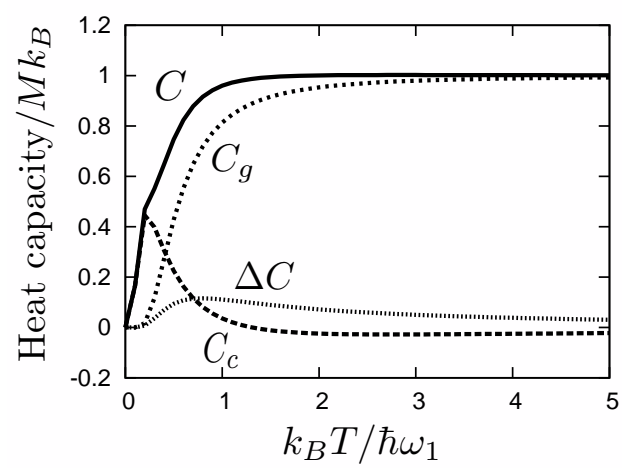

Fig. 2. Calculated temperature dependence of the heat capacities using the model consisting of $M$ independent elements. Each element takes either of the two quantum harmonic oscillators. Three contributions in eq. (24) are also plotted. The ratio of the frequencies is given by $\omega_{2} / \omega_{1}=2$. The difference in the potential bottoms is given by $\epsilon_{2}-\epsilon_{1}=-\hbar \omega_{1}$.

vanished, while $C$ and $C_{g}$ approached the classical value $k_{B}$. Note that $C_{c}$ can have negative values because it is not a heat capacity defined in an ordinary manner.

The integral of the glass heat capacity leads to the new entropy defined by eq. (14) $S_{h}$, whose values were larger than those of the configurational entropy $S_{c}$ (Fig. 1). We obtained $S_{h}=0.89 M k_{B}$ at $k_{B} T=5.0 \hbar \omega_{1}$, and $S_{c}=0.66 M k_{B}$. The difference between $S_{h}$ and $S_{c}$ was $0.23 M k_{B}$, which was $34 \%$ of the $S_{c}$ value. The values of $S_{h}$ were larger than those of $S_{c}$, because $\left\langle\Delta s_{i} \Delta e_{i}\right\rangle$ was positive at all temperatures when the present parameters were employed.

The difference between the values of $S_{h}$ and $S_{c}$ changed with the bottom of the potential. 


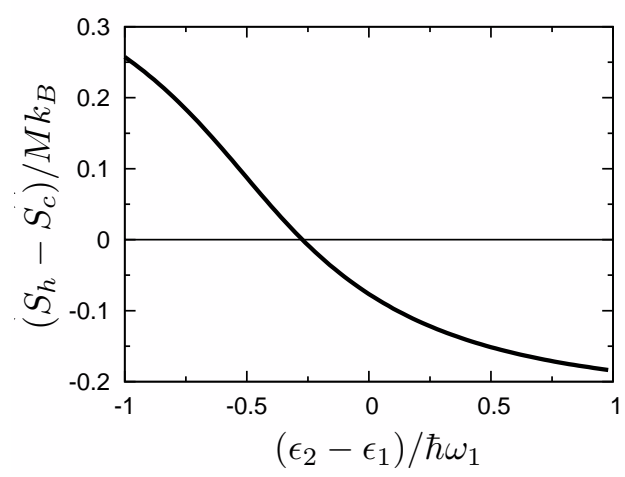

Fig. 3. Calculated $\epsilon_{2}-\epsilon_{1}$ dependence of the difference between the entropy obtained from the glass heat capacity and the configurational entropy $S_{h}-S_{c}$ at $T \rightarrow \infty$, where $\epsilon_{2}-\epsilon_{1}$ is the potential bottom difference. The model consists of $M$ independent elements, each of which takes either of the two quantum harmonic oscillators. The ratio of the frequencies is given by $\omega_{2} / \omega_{1}=2$.

The entropy difference $S_{h}-S_{c}$ was positive when the potential bottom difference $\epsilon_{2}-\epsilon_{1}$ had a large negative value. As $\epsilon_{2}-\epsilon_{1}$ increased, $S_{h}-S_{c}$ decreased to a negative value (Fig. 3).

The change in the difference between the two entropies can be explained by the correlation between the $X_{a}$-dependent energy and the $X_{a}$-dependent entropy. The $X_{a}$-dependent entropy per element $s_{1}$ in eq. (20) is larger than $s_{2}$ independently of $\epsilon_{2}-\epsilon_{1}$ when $\hbar \omega_{2}>\hbar \omega_{1}$. In addition, if $\epsilon_{2}-\epsilon_{1}$ has a large negative value, eq. (19) shows $e_{1}>e_{2}$. Then, eq. (15) shows $S_{h}>S_{c}$ because we obtain $\langle\Delta s \Delta e\rangle>0$ or $\langle\Delta S \Delta E\rangle>0$. In contrast, if $\epsilon_{2}-\epsilon_{1}$ is larger than the value of $-0.26 \hbar \omega_{1}, e_{1}<e_{2}$, so that $\langle\Delta s \Delta e\rangle<0$ or $\langle\Delta S \Delta E\rangle<0$; we obtain $S_{h}<S_{c}$.

\section{Conclusion}

The present study shows that one cannot exactly obtain the configurational entropy only from heat capacities. The configurational entropy is different from $S_{h}$ defined by eq. (14) because eq. (10) includes the term describing temperature changes in the distribution $P\left(\left\{X_{i}\right\}\right)$. Using statistical mechanics, we have obtained the results only by an assumption that degrees of freedom can be divided.

While the configurational entropy is different from the entropy obtained using the heat capacity, the difference can be expressed by the correlation between the $X_{i}$-dependent energy and the $X_{i}$-dependent entropy. Thus, the difference between $S_{c}$ and $S_{h}$ shows thermodynamic characters of liquid structures, if $\left\{X_{i}\right\}$ are the degrees of freedom expressing a liquid structure. In this case, the difference $S_{h}-S_{c}$ is positive when low-energy structures tend to have a low entropy at all temperatures. In contrast, the difference is negative when low-energy structures tend to have a high entropy.

Using a simple model, the present study also shows the possibility that the difference is too large to neglect. By the calculation using the model, we have obtained large values 
of the difference. A significant difference between $S_{c}$ and $S_{h}$ of an actual supercooled liquid can possibly be obtained, though the present model is too simple to describe actual liquids. To obtain more quantitative values of the difference, one requires quantitative theories of supercooled liquids.

The present simple model also gave an explicit example of the correlation between the $X_{i}$-dependent energy and the $X_{i}$-dependent entropy. An oscillator with a large frequency has a small value of the $X_{i}$-dependent entropy. In addition, when the bottom of the potential is low, the oscillator has a small value of the $X_{i}$-dependent energy. Thus, the correlation between the $X_{i}$-dependent entropy and energy is positive when an oscillator with the low bottom has a large frequency, so that one can obtain $S_{h}>S_{c}$ from eq. (15). In contrast, the correlation is negative or $S_{h}<S_{c}$ when an oscillator with the low bottom has a small frequency.

Here, we should note that the discrepancy observed between the experimental ${ }^{4}$ and theoretical $^{12}$ CRR sizes is not directly associated with the difference between $S_{c}$ and $S_{h}$. The method proposed by Yamamuro et al. ${ }^{4}$ shows that the CRR size is given by $S_{c}(\infty) / S_{c}(T)$, where $S_{c}(T)$ is the configurational entropy at the temperature $T$. Since the CRR size is not associated with the absolute value but the ratio, the absolute value of $S_{c}$ cannot be discussed from the CRR size. Thus, one cannot conclude that the experimental values of $S_{c}$ are larger than the theoretical values, only from the comparison of the CRR sizes. Although the present simple model allows one to calculate the ratio of entropies, more quantitative theories are required for quantitative discussions.

The present study shows a possibility of new experiments. If the absolute value of $S_{c}$ is experimentally obtained, for example, from the time scale of structural relaxation, one can obtain $\langle\Delta E \Delta S\rangle$ from the difference between $S_{c}$ and $S_{h}$. The value of $S_{h}$ can also be obtained experimentally by the method proposed by Yamamuro et al. ${ }^{4}$ If the degrees of freedom are divided by structural relaxation and fast vibrations, the positive value of $S_{h}-S_{c}$ shows that low-energy structures tend to have low entropies. The negative values show that low-energy structures tend to have high entropies.

In the new experiments, one has the difficulty arising from methods of obtaining the absolute values of $S_{c}$. It is difficult to obtain the absolute values of $S_{c}$ using the Adam-Gibbs relation. The Adam-Gibbs relation shows that $S_{c}^{-1}$ is proportional to the activation free energy in the time scale of structural relaxation. One, however, does not have methods of estimating the proportional coefficient. Thus, it is difficult to obtain the absolute values of $S_{c}$ even when the time scale of structural relaxation can experimentally be obtained.

Although the absolute values of $S_{c}$ are difficult to obtain, one can experimentally show that $S_{c}$ differs from $S_{h}$. If one observes the structural relaxation time $\tau$ and entropy $S_{h}$ at various temperatures $T$, one can plot $\ln \tau$ against $\left(T S_{h}\right)^{-1}$ using the observed data. When $S_{c}=S_{h}$, from the Adam-Gibbs relation, one finds that the plots lie on a straight line. ${ }^{23} \mathrm{In}$ 
contrast, $S_{c} \neq S_{h}$ is shown by the deviation from the line.

Using the plot, the difference between $S_{c}$ and $S_{h}$ has been obtained by some experiments on molecular liquids ${ }^{23,24}$ and orientational-disordered crystals. ${ }^{25}$ In particular, a large deviation was observed in cyanoadamantane, ${ }^{25}$ which has a glass transition associated with its orientational disorder. ${ }^{26,27}$ One can obtain the correlation between the $X_{i}$-dependent energy and the $X_{i}$-dependent entropy by a detailed study of the deviation.

\section{Acknowledgment}

This work was supported in part by the Grant-in-Aid for Scientific Research on Priority Area and for Scientific Research (c) 19540405 and 22540400 from the Japanese Ministry of Education, Culture, Sports, Science and Technology. 


\section{References}

1) A. Yoshimori and T. Odagaki: J. Phy. Soc. Jpn. 74 (2005) 1206.

2) T. Odagaki, T. Yoshidome, A. Koyama, and A. Yoshimori: J. Non-Cryst. Solids 352 (2006) 4843.

3) T. Odagaki and A. Yoshimori: J. Non-Cryst. Solids 355 (2009) 681.

4) O. Yamamuro, I. Tsukushi, A. Lindqvist, S. Takahara, M. Ishikawa, and T. Matsuo: J. Phys. Chem. B 102 (1998) 1605.

5) K. Takeda, O. Yamamuro, I. Tsukushi, T. Matsuo, and H. Suga: J. Mol. Struct. 479 (1999) 227.

6) O. Yamamuro, Y. Oishi, M. Nishizawa, and T. Matsuo: J. Non-Cryst. Solids 235 (1998) 517.

7) O. Yamamuro, Y. Minamimoto, Y. Inamura, S. Hayashi, and H. Hamaguchi: Chem. Phys. Lett. 151 (1988) 47.

8) M. Cardenas, S. Franz, and G. Parisi: J. Chem. Phys. 110 (1999) 1726.

9) M. Mézard and G. Parisi: J. Chem. Phys. 111 (1999) 1076.

10) B. Coluzzi, M. Mézard, G. Parisi, and P. Verrocchio: J. Chem. Phys. 111 (1999) 9039.

11) G. Adam and J. H. Gibbs: J. Chem. Phys. 43 (1965) 139.

12) T. Yoshidome, A. Yoshimori, and T. Odagaki: Phys. Rev. E 76 (2007) 021506.

13) J. Matsui, private communication.

14) T. Odagaki and T. Ekimoto: J. Non-Cryst. Solids 353 (2007) 3928.

15) T. Ekimoto, T. Odagaki, and A. Yoshimori: Conf. Proc. Complex Systems 982 (2008) 211.

16) T. Yoshidome, A. Yoshimori, and T. Odagaki: J. Phy. Soc. Jpn. 75 (2006) 054005.

17) T. Yoshidome, T. Odagaki, and A. Yoshimori: Phys. Rev. E 77 (2008) 061503.

18) T. Tao, A. Yoshimori, and T. Odagaki: Phys. Rev. E 64 (2001) 046112.

19) T. Tao, A. Yoshimori, and T. Odagaki: Phys. Rev. E 66 (2002) 041103.

20) T. Odagaki, T. Tao, and A. Yoshimori: J. Non-Cryst. Solids 307 (2002) 407.

21) T. Odagaki, T. Yoshidome, T. Tao, and A. Yoshimori: J. Chem. Phys. 117 (2002) 10151.

22) T. Tao, T. Odagaki, and A. Yoshimori: J. Chem. Phys. 112 (2005) 044504.

23) S. Takahara, O. Yamamuro, and T. Matsuo: J. Phys. Chem 99 (1995) 9589.

24) O. Yamamuro, M. Ishikawa, I. Tsukushi, and T. Matsuo: AIP Conference Proc. 469 (1999) 513.

25) O. Yamamuro, M. Ishikawa, I. Kishimoto, J.-J. Pinvidic, and T. Matsuo: J. Phy. Soc. Jpn. 68 (1999) 2969.

26) M. Descamps, C. Caucheteux, G. Odou, and J. L. Sauvajol: J. Physique Lett. 45 (1984) L719.

27) H. Takakura and N. Achiwa: J. Korean Phy. Soc. 29 (1996) S533. 